\title{
Optical Aberration Correction via Phase Diversity and Deep Learning
}

\author{
Anitha Priya Krishnan, ${ }^{1}$ Chinmay Belthangady, ${ }^{1}$ \\ Clara Nyby, ${ }^{2}$, Merlin Lange ${ }^{1}$, Bin Yang ${ }^{1}$ and Loic A. Royer ${ }^{1, *}$ \\ ${ }^{1}$ Chan Zuckerberg Biohub, San Francisco, CA 95158, USA \\ ${ }^{2}$ Department of Chemistry, Stanford University, Stanford, CA 94305, USA \\ *loic.royer@czbiohub.org
}

\begin{abstract}
In modern microscopy imaging systems, optical components are carefully designed to obtain diffraction-limited resolution. However, live imaging of large biological samples rarely attains this limit because of sample induced refractive index inhomogeneities that create unknown temporally variant optical aberrations. Importantly, these aberrations are also spatially variant, thus making it challenging to correct over wide fields of view. Here, we present a framework for deep-learning based wide-field optical aberration sensing and correction. Our model consists of two modules which take in a set of three phase-diverse images and (i) estimate the wavefront phase in terms of its constituent Zernike polynomial coefficients and (ii) perform blind-deconvolution to yield an aberration-free image. First, we demonstrate our framework on simulations that incorporate optical aberrations, spatial variance, and realistic modelling of sensor noise. We find that our blind deconvolution achieves a 2-fold improvement in frequency support compared to input images, and our phase-estimation achieves a coefficient of determination $\left(r^{2}\right)$ of at least $80 \%$ when estimating astigmatism, spherical aberration and coma. Second, we show that our results mostly hold for strongly varying spatially-variant aberrations with a 30\% resolution improvement. Third, we demonstrate practical usability for light-sheet microscopy: we show a $46 \%$ increase in frequency support even in imaging regions affected by detection and illumination scattering.
\end{abstract}

\section{Introduction}

High-resolution optical imaging is of great importance in fields like astronomy, microscopy, and biomedical imaging. However, the resolution of the acquired images is often limited by system as well as sample-induced optical aberrations. While system aberrations are caused by limitations in optical design, sample-induced aberrations may be caused by complex refractive index changes within the sample that vary in both space and time. One such example is the live imaging of fluorescently labelled embryos using light-sheet microscopy [1,2]. Given a known and invariant Point Spread Function (PSF), a popular solution for addressing system aberrations is to apply deconvolution algorithms such as Richardson-Lucy (RL) [3] which can be extended for multi-view imaging $[4,5]$. If the PSF is unknown, blind deconvolution (BD) algorithms can jointly estimate the image and PSF [6]. Another solution of choice is Adaptive Optics (AO) which implements wavefront sensing (i.e. measures deviation from a planar wavefront) and correction in hardware by means of a Shack-Hartmann sensor and deformable mirror, respectively. While highly effective, corrections afforded by AO are generally only valid over a small field-of-view, or require prohibitively complex hardware setups capable of multi-conjugate correction [7]. In contrast, other phase retrieval approaches can estimate lower order aberrations across a wide field-of-view from multiple images acquired with known phase offsets [8], and can jointly estimate object and aberrations [9].

With the success of Convolutional Neural Networks (CNNs) in performing various image-based tasks (see [10] for a comprehensive review), CNN models have been developed for deconvolution and wavefront sensing. Some of these approaches use a blur kernel that includes focus and 
astigmatism only or use larger kernels that are not physically realisable in a microscope $[11,12]$ and typically do not take advantage of additional information from phase-diverse images. Other approaches model the image estimation part with a CNN and use a deconvolution module [11,13] to extract the PSF, or use existing CNN architectures such as ResNet and Inception to regress Zernike coefficients $[12,14,15]$, and use iterative Richardson-Lucy to estimate the image but not both.

Here we present a CNN approach for blind deconvolution and phase estimation, both of which take advantage of phase-diverse image acquisitions. The key aspects of our work are: (i) the extraction of 3D convolutional features from phase-diversity stacks - so as to capture axial extent of PSF and thus facilitate deconvolution; (ii) the estimation of up to $4^{\text {th }}$ order Zernike coefficients including astigmatism, coma and trefoil; (iii) the training of an end-to-end framework for joint estimation of the PSF and deconvolved image; (iv) a demonstration of correction on spatially varying aberrations; (v) the application on a phase-diverse dataset acquired on a light-sheet microscope - to better understand the performance and limits of our approach.

Related Work. Earlier CNN models for deconvolution unroll the iterative algorithm and solve it as a nonlinear regression problem. Zhang et. al. developed a cascaded Fully Convolutional Neural Network (FCNN) to address the effect of noise and sensitivity to image-priors in non-blind deconvolution of natural images. The model denoised the vertical and horizontal gradients which were used as image priors to an iterative deconvolution algorithm [11]. Schuler et.al. used a similar multistage model for blind deconvolution of natural images with each stage consisting of a feature extraction, kernel estimation, and image estimation module. The resulting model outperformed conventional approaches for small and medium size blur kernels, but failed for larger kernels [13]. Recently, Shajkofci et. al. fine-tuned Alex-net and Res-net to estimate optical aberrations in microscopy images, and performed semi-blind deconvolution using total variation $R L$ [12]. On a related note, Paine et.al. trained an Inception model and its variant for estimating a good initial guess of the wavefront [14]. and Nishizaki et.al. used iterative wavefront sensing approaches in combination with an image pre-conditioner for overexposure, defocus, and scatter [15].

\section{Theory}

Wavefront parameterization. Wavefront phase is commonly modeled as a series expansion of Zernike polynomials [16], which are orthogonal polynomials on the unit circle:

$$
\begin{aligned}
& Z_{n}^{m}(\rho, \theta)=R_{n}^{m}(\rho) \cos m \theta \\
& Z_{n}^{-m}(\rho, \theta)=R_{n}^{m}(\rho) \sin m \theta
\end{aligned}
$$

A double indexing scheme is used, where index $n$ represents the highest power of the polynomial for the radial component $(\rho: 0-1)$, index m represents the frequency of the azimuthal $\operatorname{component}(\theta$ : $0-2 \pi$ ), and $R_{n}^{m}$ is the radial component of the Zernike polynomials [12]. Wavefront aberrations are described as the sum of Zernike modes $Z_{j}$ multiplied by their coefficient $C_{j}$ indexed by the Zernike mode number $\mathrm{j}$ :

$$
W(\rho, \theta)=\sum_{j} C_{j} Z_{j}(\rho, \theta) \quad \text { where } \quad j=\frac{n(n+2)+m}{2}
$$

Hence, the Zernike coefficient vector $Z=\left[C_{j}\right]$ is sufficient to fully characterise the wavefront.

Image formation. At a focus distance $\epsilon$, the point spread function is related to wavefront distortion by:

$$
P S F(\epsilon)=\left|\mathcal{F}\left(P(\rho, \theta) e^{i W(\rho, \theta)+i d(\rho, \epsilon)}\right)\right|^{2}
$$


Where, $\mathrm{P}$ is the pupil function and $\mathcal{F}$ is the Fourier transform. The defocus-only phase diversity [8] under paraxial approximation is given by:

$$
d(\rho, \epsilon)=\frac{\epsilon \mathrm{NA}^{2} \rho^{2}}{2 \lambda}
$$

where NA is the numerical aperture, $\lambda$ is the optical wavelength, and $\epsilon$ is the focus distance. For simplicity our model assumes that light is emitted from a single plane within the sample - as if illuminated by an infinitely thin and extended light-sheet. In what follows we consider an objective with a numerical aperture of $N A=0.8$ and a pixel size of $0.406 \mu \mathrm{m}$.

Problem statement. The observed aberrated image is given by: $I_{0}=I * P S F(0)$ where $I$ is the non-aberrated image. In addition to optical aberrations, these images are also degraded by shot noise and electronic noise. For the purpose of phase retrieval, we also acquire two additional slightly out-of-focus phase-diverse images: $I_{-1}=I * P S F(-\epsilon)$ and $I_{+1}=I * P S F(+\epsilon)$ where $\epsilon$ is the absolute defocus. While more than three phase-diverse images can be used, one should also consider the practicality and cost in time of acquiring more than three images. Our goal is, given the images $I_{0}, I_{-1}$, and $I_{+1}$, to obtain an estimate $I^{\prime}$ of the the true object image $I$ and the corresponding estimate $Z^{\prime}$ of the true Zernike coefficients $Z$.

Implicitly learned image prior. Classical iterative solutions $[8,17]$ for estimating the true object image $I$ impose analytical constrains - such as e.g. non-negativity - on the structure of solutions $I^{\prime}$. The strength of generality of classical - non deep-learning - methods is also a weakness because for specific imaging applications strong solution priors exist that can substantially improve reconstruction quality. One of the key advantages of CNNs is their ability to learn both the inverse function but also an implicit prior on solutions $[10,18]$. In this work we focus our attention on light-sheet microscopy images of fluorescently labelled nuclei in developing zebrafish (D. rerio) and fruit fly (D. melanogaster) embryos. Imaging within such large embryos is challenging because of sample induced aberrations that rapidly degrade image quality for deep imaging planes $(>50 \mu \mathrm{m})$. Yet, these images are highly stereotypical: while exhibiting some variance, cell nuclei have a typical size, shape and spatial distribution, and thus offers an opportunity for improved image deconvolution and phase estimation using deep learning.

\section{Methods}

Sample preparation. Mounting and imaging of zebrafish embryos were handled in accordance with the University of California San Francisco guidelines and were approved by the Institutional Animal Care and Use Committee. Imaging experiments were performed on the zebrafish transgenic line h2afva:h2afva-mCherry. After dechorionation, embryos were mounted in $0.8 \%$ low melting point agarose inside a $1.5 \mathrm{~mm}$ inner diameter glass capillary. After agarose solidification, the agarose section containing the embryo is extruded from the glass capillary with a plunger. The sample is then placed in a custom-made multi-view light-sheet microscope [2] after filling the imaging chamber with E3 fish medium.

Microscopy. We use images of developing zebrafish and fruit fly embryos previously acquired on a light-sheet microscope [2] for the simulations described below, as well as newly acquired phase-diverse zebrafish images for evaluation. Imaging is done with Nikon $16 \times 0.8 \mathrm{NA}$ waterdipping objectives and an Orca Flash $4.0 \mathrm{sCMOS}$ camera resulting in a pixel size of $0.406 \mu \mathrm{m}$. we illuminate the sample with a $561 \mathrm{~nm}$ laser light-sheet and filter detection using a 610/75 bandpass filter. Exposure is set to $20 \mathrm{~ms}$. 
Simulated training data. For each dataset (zebrafish and fruit fly), the images are first denoised using Noise2Self [19], then image tiles of size $256 \times 256$ pixels are randomly selected, and then normalised to have intensity values within $[0,1]$. The tiles are convolved with PSF kernels of size $31 \times 31$ pixels to generate aberrated images for training. For simplicity we assume that fluorescent light is emitted from an infinitely thin slab of material co-planar with the detection plane. To compute the PSF images, we use Eq. 1. To model the sample induced aberrations, Zernike coefficients of orders 2,3 and 4 are sampled from a uniform random distribution between $[-1,1]$. The sampled Zernike coefficients are multiplied by a bi-exponential decay term that adjusts the contributions of the various Zernike orders. We do not include lateral image shifts $\left(Z_{1}^{-1}, Z_{1}^{1}\right)$ and focus $\left(Z_{2}^{0}\right)$ since these can be corrected with registration and auto-focus methods [20]. A defocus term is added and subtracted from these Zernike coefficients to obtain out-of-focus PSFs to generate the phase-diverse images $I_{0}, I_{-1}$, and $I_{+1}$. we use a defocus $\epsilon$ of $+/-2 \mu \mathrm{m}$ Poisson noise $(n=150)$ and Gaussian noise $(\sigma=0.001)$ are applied to the blurred tiles to simulate both realistic sensor and photon shot noise.

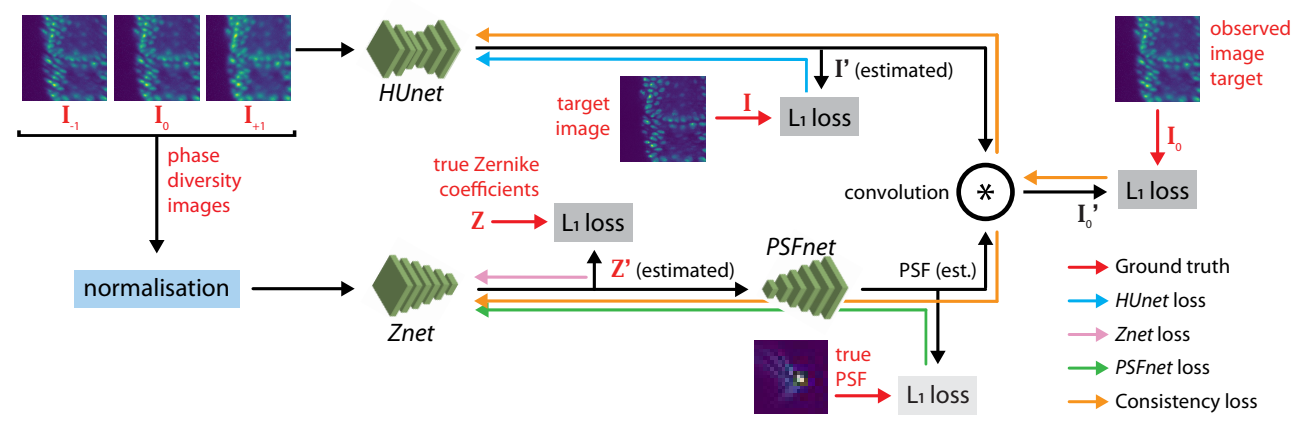

Fig. 1. Model Overview. The two networks HUnet and Znet compute the deconvolved image $I^{\prime}$ and Zernike coefficients $Z^{\prime}$, respectively, from phase-diverse images $I_{-}, I_{0}$, and $I_{+}$. PSFnet is a differentiable model used for enforcing self-consistency that computes the PSF from a corresponding Zernike vector $Z^{\prime}$. We show all four losses considered and corresponding gradient paths.

Model overview. As shown in Fig. 1 our model consists mainly of a deconvolution model HUnet and phase estimation model Znet. An additional pre-trained differentiable model PSFnet is used for generating a PSF image from the predicted Zernike coefficients - this model is used for enforcing consistency between the deconvolution and phase estimation. All models are implemented in python using the Deep-Learning library PyTorch. In the following paragraphs we give architectural details on each model.

HUnet. This network is a 3D-2D hybrid version of the U-net [21], where the down-sampling arm uses $3 \mathrm{D}$ convolutions with $3 \times 3 \times 3$ kernels and the up-sampling arm uses $2 \mathrm{D}$ convolutions implemented via anisotropic $1 \times 3 \times 3$ kernels (Fig. 2a). The use of 3D convolutions allows the network to identify 3D Patterns within the three images $\left(I_{-}, I_{0}\right.$, and $\left.I_{+}\right)$, which together are equivalent to a defocus stack. Skip connections consist of $N \times 1 \times 1$ convolutions (with no zero-padding) followed by batch normalisation, where $N$ is the number of phase diverse images and batch normalization. We use nearest-neighbour interpolation for up-sampling. This network, with 82 layers and 1.23 million trainable parameters, takes a stack of three images $I_{0}, I_{-1}$, and $I_{+1}$ and predicts the deconvolved image $I^{\prime}$. 
Znet. This network predicts Zernike coefficients $Z$ from the three phase-diverse images $I_{0}, I_{-1}$, and $I_{+1}$. In a manner similar to the HUnet, we use $3 \mathrm{D}$ convolutions with $3 \times 3 \times N(N$ : number of phase diverse images, $N=3$ ) kernels, down-sampling, and residual connections to convert the phase-diverse input image tensor of size $3 \times 256 \times 256$ to an estimated Zernike vector $Z^{\prime}$ of size $1 \times 1 \times 12$ (see Fig. 2b). The Znet has 76 layers and 14.15 million parameters.

PSFnet. This network provides a differentiable and numerically stable learned approximation of the function that computes the PSF from estimated Zernike coefficients $Z^{\prime}$ (see Eq. 1). It consists of four blocks with each block consisting of transposed convolution for up-sampling, batch-normalization, ReLU nonlinearity and dropout layers [22] (Fig. 2c). The final block consists of convolution and hyperbolic tangent activation layers. The network has 13 layers and 2.85 million trainable parameters.

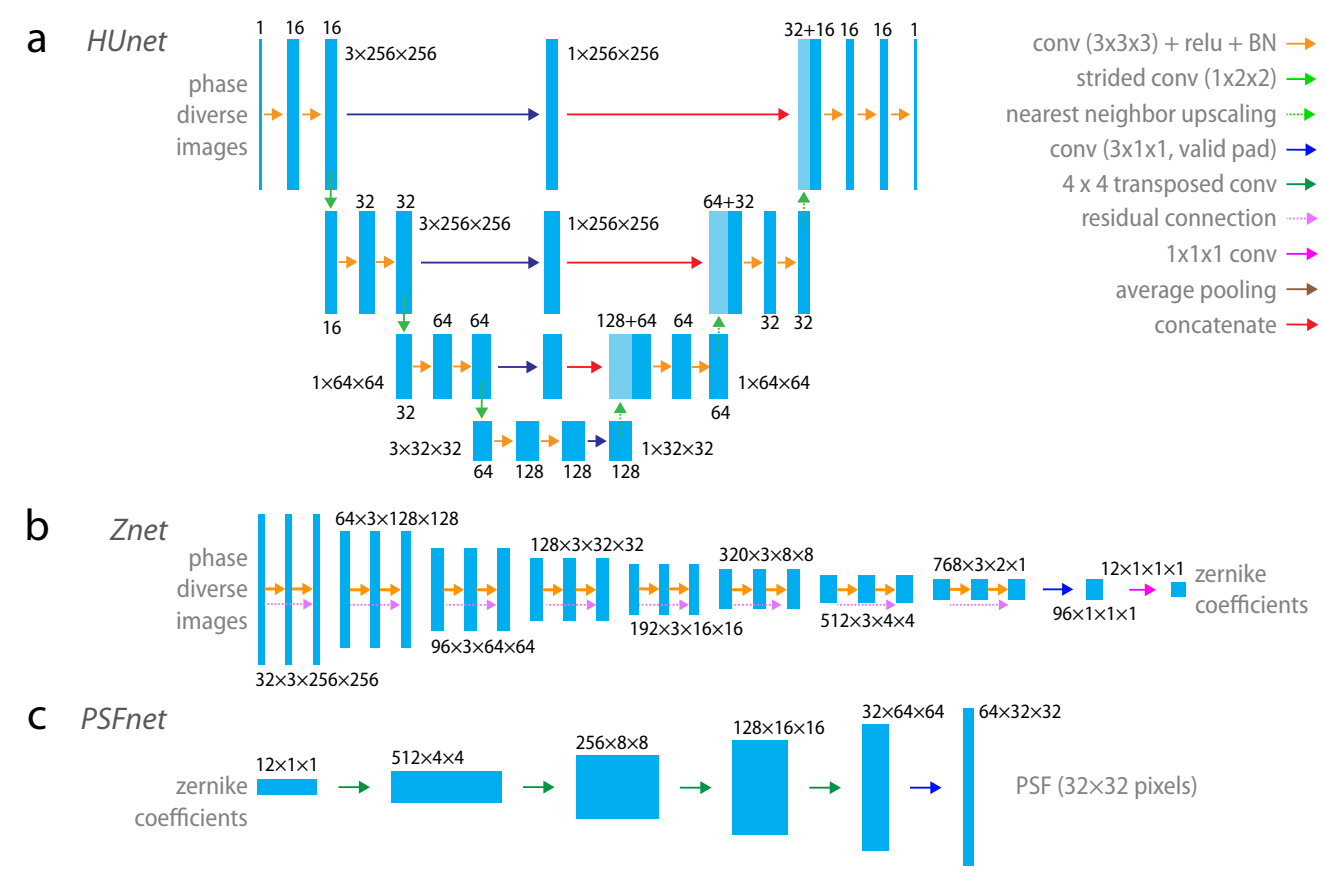

Fig. 2. Detailed model architectures for (a) HUnet, (b) Znet, and (c) PSFnet. HUnet is a hybrid 3D-2D UNet [23] that receives three phase diverse images $I_{-}, I_{0}$, and $I_{+}$ as a single stacked $3 \mathrm{D}$ image, and returns a single $2 \mathrm{D}$ deconvolved image $I^{\prime}$. Znet is made of 8 residual convolutional blocks that take the three phase-diverse images and returns a single vector of 12 Zernike coefficients. PSFnet takes a vector of 12 Zernike coefficients and returns the PSF sampled on a $32 \times 32$ image. Note: these architectures can be easily be adapted to accommodate an arbitrary number $N$ of phase diverse inputs.

Joint training. We hypothesise that training both HUnet and Znet jointly and enforcing consistency between the deconvolved image $I^{\prime}$ and the estimated Zernike coefficients $Z^{\prime}$ will encourage the networks to truly learn image deconvolution and Zernike coefficient estimation and possibly prevent the models from over-fitting on image features. Four losses are used for joint training: $L_{1}$ loss between $I$ and $I^{\prime}$, between $Z$ and $Z^{\prime}$, between the output of PSFnet and the 
true PSF (PSFnet weights are held fixed but gradients can pass through Znet), and a consistency loss. The deconvolved image $I^{\prime}$ from HUnet is convolved with the predicted PSF to infer a reconstructed aberrated image $I_{0}^{\prime}$ from both $I^{\prime}$ - computed by HUnet, and $Z^{\prime}$ - computed by Znet. The $L_{1}$ loss between this predicted blurred image $I_{0}^{\prime}$ and the center image $\left(I_{0}\right)$ provides the consistency loss that is backpropagated to update parameters of both HUnet and Znet (Fig. 1).

Training parameters. HUnet and Znet are trained using $L_{1}$ loss, the Adam optimiser, and a learning rate of $10^{-4}$. We use a batch size of 16 and the models are trained for 300 epochs with a $70-15-15$ split for train, validation and test sets. To prevent overfitting we use a dropout of 0.2 and use a random subset of tiles for training in every epoch. Training takes 17 hours for HUnet and 24 hours for Znet (NVIDIA Titan X). PSFnet is trained on a separate set of 10000 pairs of Zernike coefficients (sampled in the same manner as previously described for the HUnet training data) and analytically computed PSF images of size $31 \times 31$ pixels. The PSFs are normalized to have an area under the curve of one. The PSFnet achieves an SSIM of 0.967 between the target and predicted PSF images.

Iterative non-blind deconvolution. We compare the performance of our approach against three iterative non-blind deconvolution algorithms: (i) Richardson-Lucy Total Variation (RLTV) which augments Richardson-Lucy's approach [24] with a TV prior [25], (ii) Iterative Constraint Tikhonov-Miller (ICTM) which minimizes a Tikhonov functional and enforces non-negativity at each iteration [26], and (iii) Bounded-Variable Least Squares (BVLS) [27]. For all three approaches we use DeconvolutionLab2 implementations [27].

Evaluation metrics. Mean structural similarity (SSIM) [28], mean coefficient of determination $\left(r^{2}\right)$ over the test set are used as metrics for evaluating HUnet performance. Mean coefficient of determination and mean absolute error (MAE) for each predicted Zernike coefficient is used for evaluating Znet performance.

\section{Results}

Deconvolution performance. Overall, our blind deconvolution model (HUnet) trained on the zebrafish dataset performed slightly better than that trained on fly dataset (SSIM of 0.421 for fly versus 0.932 for zebrafish, see Table 1) which might be attributed to the larger nuclei found in the zebrafish dataset. Importantly, both models generalised well when trained on one dataset and applied onto the other - with models trained on zebrafish generalizing marginally better (Table 2).

Comparison with state-of-the-art iterative non-blind deconvolution. As stated previously, We compared our phase diversity blind deconvolution against classical deconvolution non-blind algorithms to which we provide the true PSF. Of the three deconvolution methods compared, ICTM performs best with a mean SSIM of 0.317 on the fly dataset and 0.556 on the zebrafish dataset, which is an improvement of approximately $20 \%$ and $17 \%$ compared to blurry input images for the respective datasets (Table 1). However, our deconvolution model ( HUnet) achieved a mean SSIM of 0.421 on the fly dataset and of 0.932 on the zebrafish dataset - an improvement of approximately $60 \%$ and $95 \%$ compared to the blurry input images (Table 2). The better performance of HUnet compared to non-blind deconvolution is most likely due to its ability to implicitly learn a strong reconstruction prior from the stereotypy of nuclei images - i.e. from the repetitive features found in nuclei images. 
Frequency domain resolution analysis and comparison. As shown in Fig. 3 our HUnet deconvolution model recovers noise-free deconvolved images. Detailed image frequency analysis shows that for low frequencies (red triangle marker, features above 5 pixels) the performance of HUnet is comparable to that of the best performing deconvolution (ICTM), but for higher spatial frequencies (features of scale below 5 pixels), HUnet outperforms ICTM by rejecting the noise and in-filling lost frequency details with a very natural-looking apodization. In contrast, ICTM restored images show strong artifacts, which as shown in Fig. 3 have their origin in erroneous high frequency restitution (see black triangle marker). Using Mizutani's single image resolution estimate [29] we can quantify that improvement further: we find that HUnet deconvolved images have 2-fold wider frequency support than the best focussed input image: 1.99 and 2.34 for fly and zebrafish, respectively. Despite the artefacts mentioned previously, ICTM deconvolution performs well but still underperforms compared to HUnet: 1.52 and 1.89 fold improvement in frequency support for fly and zebrafish, respectively. Paradoxically, and yet as expected, HUnet exhibits a wider frequency support than the ground truth itself - this is because of (i) the denoising and frequency in-painting at high frequencies, (ii) the nuclei prior is learned over the whole training set based on real microscopy images with a wide distribution of sharpness which affords opportunities for further sharpening.

Spatially-variant blind deconvolution. In-toto live imaging of developing embryos involves imaging large fields of view of up to $800 \mu \mathrm{m}$. Almost certainly, the sample's varying refractive index composition is not constant over the field of view [30] which leads us to consider the problem of deconvolving images with spatially varying optical aberrations. Tackling spatial variance is challenging for standard wavefront correction approaches because corrections are necessarily limited to a small region of the field of view [30]. In contrast, blind deconvolution possibly informed by phase information - can address this problem. First, wee simulate spatial variance by varying the PSF over tiles of dimensions $32 \times 32$. At inference time, for Znet, we simply break large images into smaller tiles $(256 \times 256)$. For HUnet we rely on translation invariance. The results are shown in Fig. 4. Deconvolution with HUnet produces sharper outputs with overall $33 \%$ wider frequency support. Similarly, Znet predictions seem to match the expected behaviour of predicting the average PSF over the tile - however, in some cases (yellow inset) the predicted PSF is incorrect. These results suggest that for estimating spatially varying aberrations, training should be best done directly on a variant dataset.

Importance of phase diversity. We found very similar deconvolution performance for HUnet trained with and without phase diversity (SSIM reduction of 0.017 for fly and 0.010 for zebrafish, see Table 1) suggesting that the implicit phase information is either not used or not necessary to achieve deconvolution. Our interpretation is that the implicitly learned image prior - cell nuclei - is strong enough, rendering the phase information mostly superfluous. This encouraging result suggests that for moderate optical aberrations, blind deconvolution of stereotypical images does not require phase information. This is in agreement with previous results on isotropy restoration $[18,31]$ that showed that axial deconvolution is possible in the absence of phase information for images that have strong stereotypy. However, for more severe aberrations and noisier acquisitions, phase retrieval - as proposed here or otherwise - in concert with wavefront correction is likely required for good results.

Aberration estimation. The phase estimation model Znet performed reliably in predicting astigmatism $\left(Z_{2}^{-2,2}\right)$, coma and trefoil aberrations $\left(Z_{3}^{-3,-1,1,3}\right)$, as well as spherical aberration $\left(Z_{4}^{0}\right)$, but less so for quadrafoil and secondary astigmatism (Fig. 5). In contrast to the deconvolution model (HUnet), the aberration estimation model (Znet) trained on fly images generalized better to zebrafish images (5\% vs $11 \%$ performance reduction) suggesting less reliance on nuclei image 

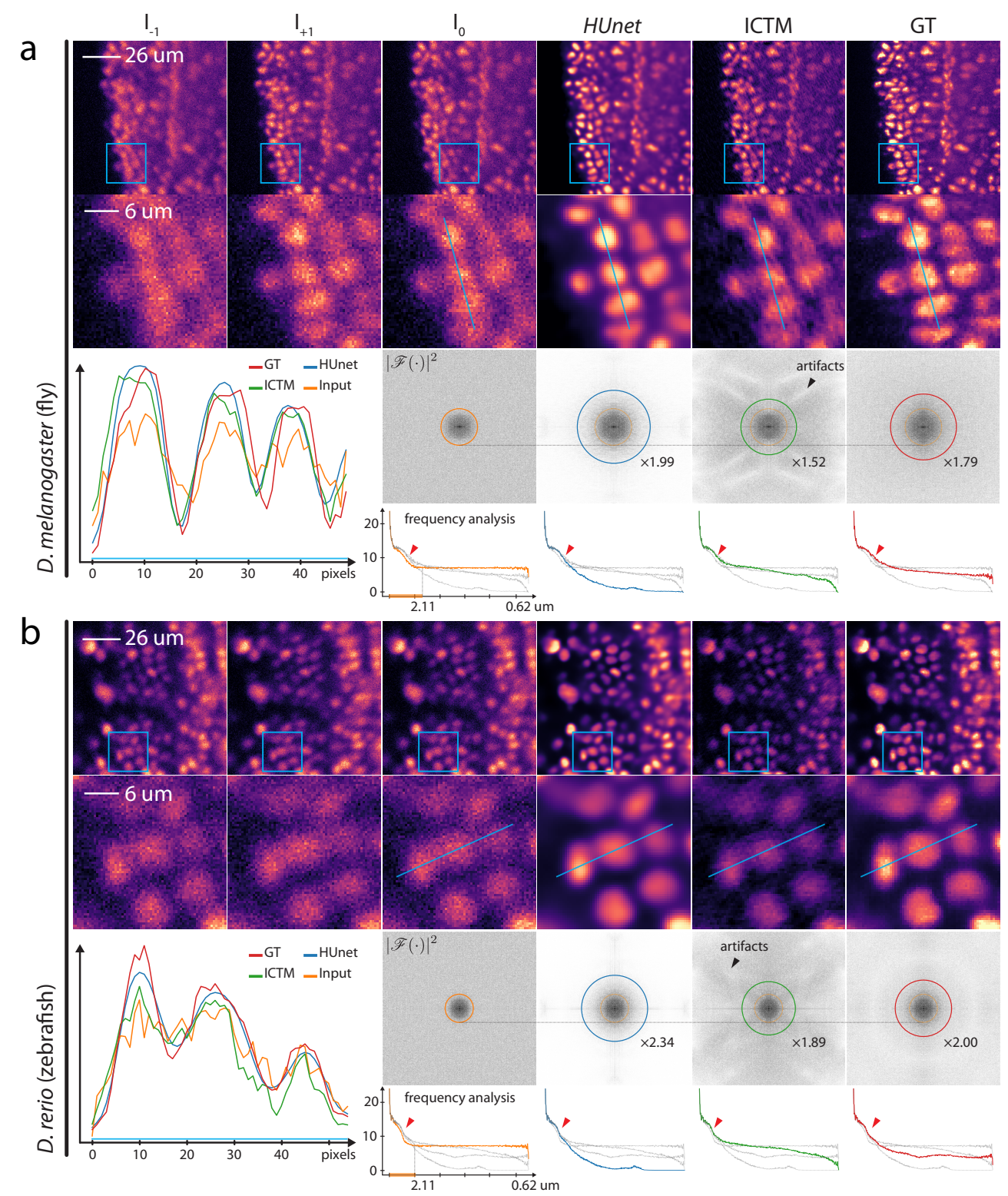

Fig. 3. Comparison between phase-diversity based HUnet deconvolution (3 image input $\left.I_{-1}, I_{0}, I_{+1}\right)$, non-blind ICTM deconvolution ( $I_{0}$ as input), and ground truth, for both (a) D. melanogaster (fly) and (b) D. rerio (zebrafish) embryo images. In addition, line profiles and Fourier frequency analysis are shown for aiding comparison of results. Quantitatively, HUnet deconvolved images have a wider frequency (2.3 fold) support and a low noise floor compared to the input images (according to Mizutani's single image resolution estimate [29]). 


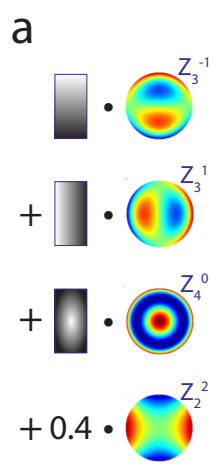

b $\quad$ I

$I_{+1}$

$I_{0}$

HUnet

GT
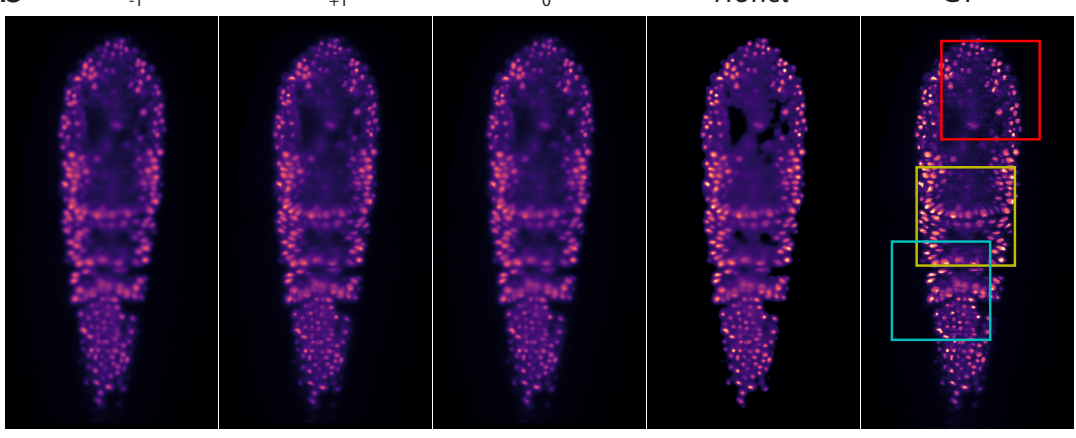

C Znet PSF

true PSF d Io

HUnet

GT

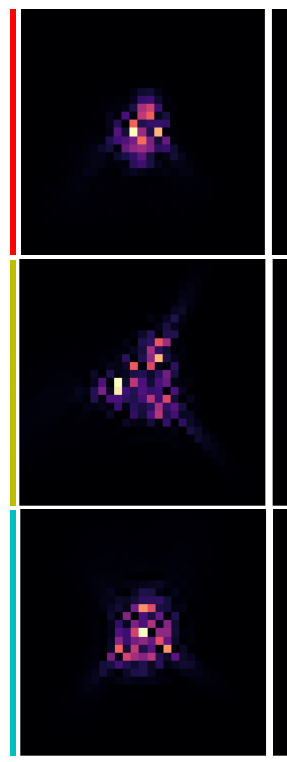

e I

$|\mathscr{F}(\cdot)|^{2}$

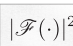

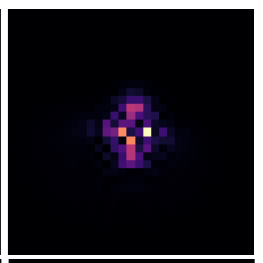
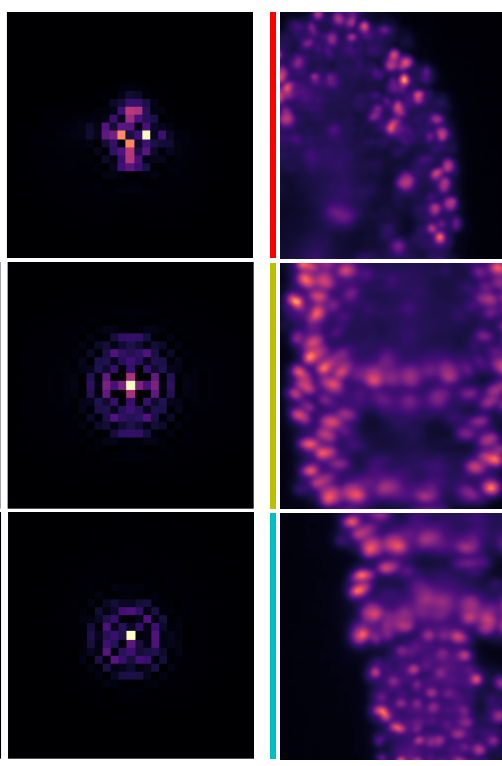

HUnet
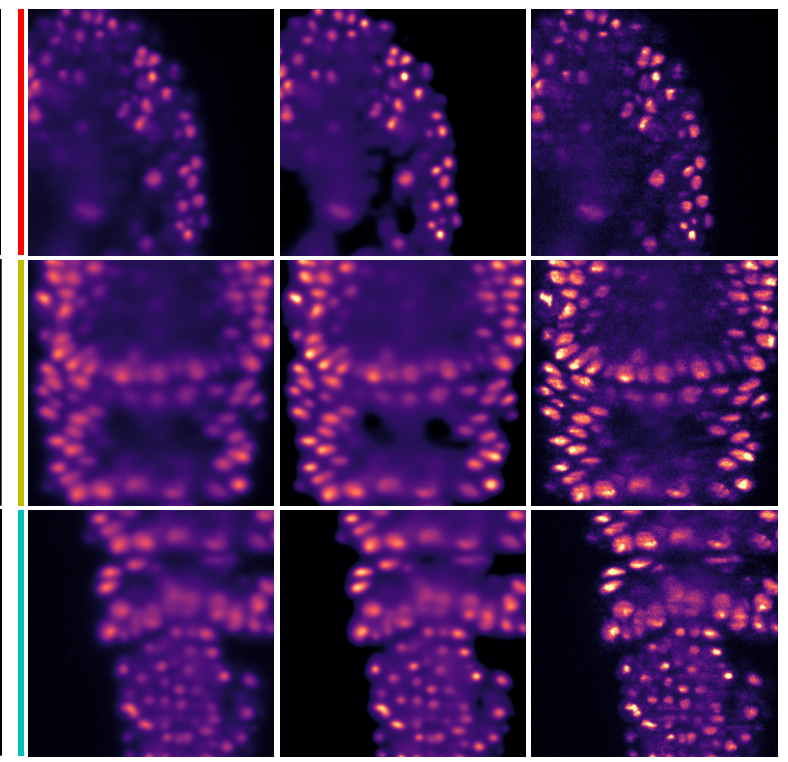

GT
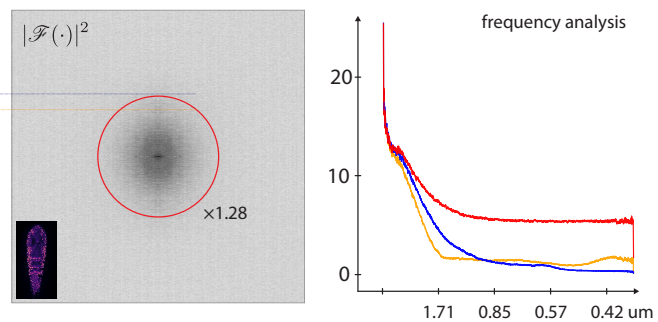

Fig. 4. Performance of HUnet and Znet for spatially varying aberrations. (a) Pictorial description of the spatially variant aberrations used for evaluation. Here we have the sum of primary spherical $\left(Z_{4}^{0}\right)$ varying with a quadratic profile, vertical $\left(Z_{3}^{-1}\right)$ and horizontal $\left(Z_{3}^{+1}\right)$ coma varying linearly along $Y$ and $X$, respectively, and constant astigmatism $\left(Z_{2}^{2}\right)$. (b) phase-diversity stack $\left(I_{-1}, I_{+1}\right.$, and $\left.I_{0}\right)$ used as input. (c) Predicted versus true PSFs. The PSFs shown here are the average of the spatially varying PSFs within each tile. (d) Expanded images for insets in (a) with the red inset along row 1, yellow along row 2 and cyan along row 3. (e) Frequency analysis shows that HUnet deconvolution frequency support is comparable to that of the ground truth, only with less noise at high frequencies. 
priors and actual utilisation of the phase diversity information (Table 3). As expected, and in contrast to our deconvolution model, aberration estimation (Znet) models trained without phase-diversity completely fail to generalise to the test set. In the best case, these models over-fit to the training set in the absence of dropout regularisation.

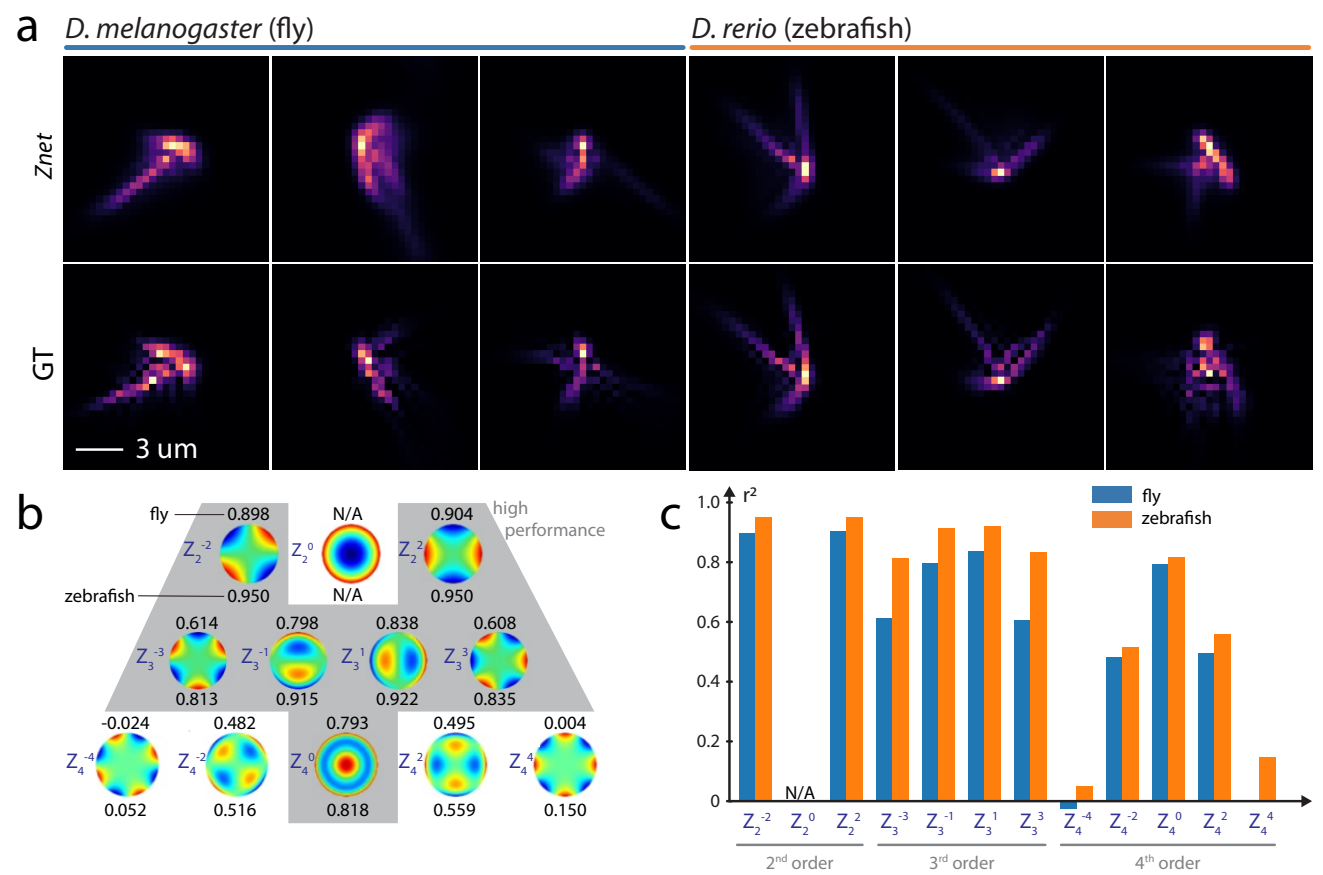

Fig. 5. (a) Znet Example PSF predictions versus corresponding ground truth PSFs. (b) Znet model performance per coefficient on fly (top) and zebrafish (bottom) datasets. The coefficient of determination $\left(r^{2}\right.$, within $\left.[0,1]\right)$ is given for each Zernike coefficient. Gray background area identifies coefficients for which model performance is high (above 80\%). Defocus is not considered as we assume in-focus imaging. (c) Bar chart showing that Zernike coefficients with high angular meridional frequency are difficult to predict - for example $Z_{4}^{-4}$ and $Z_{4}^{4}$.

Application to light-sheet acquisitions. After promising results on simulations, we wanted to test our deconvolution model (HUnet) on images acquired on a light-sheet microscope - with all the imperfections and non-idealities that this entails. In light of these circumstances, our results are promising: Fig. 6 shows an overall $46 \%$ increase in resolution as well as suppression of all high-frequency noise - a typical and beneficial side-effect of CNN inference. Yet, for deeper imaging regions (lower panels in Fig. 6) our model only marginally improves image quality because of the limits of our assumptions. Indeed, our simulation model implicitly assumes (i) an illumination confined to an infinitely thin light-sheet and (ii) no scattering on both illumination and detection paths. While overall performance is satisfactory for images taken at shallow imaging depths within the zebrafish embryo, for deeper regions, we observe strong out-of-focus light and background due to a thick light-sheet and detection scattering - the resulting images, while apparently sharper, should be treated with caution because the input images strongly violate our assumptions. Overall, our observations reinforce the notion that low-order aberrations (as corrected by HUnet) are not the only - or even dominant - cause of image quality degradation 
in light-sheet imaging of large samples such as zebrafish embryos [30]. Instead, higher-order aberrations and scattering on both illumination and detection paths account for most of the image quality degradation.

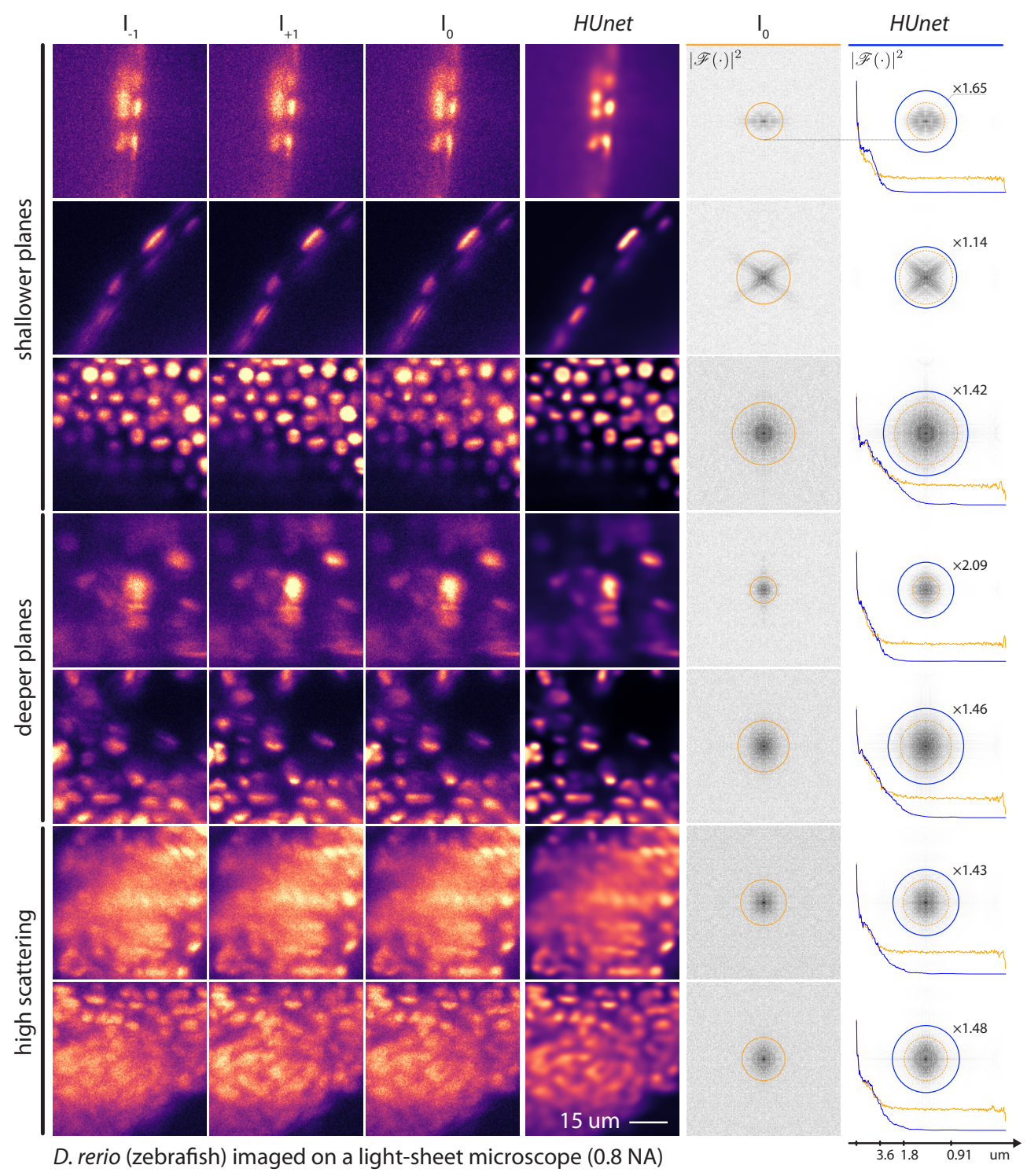

Fig. 6. Applying HUnet deconvolution on phase-diverse acquisitions from a light-sheet microscope (0.8 NA, 16×, pixels 0.406). Applying HUnet to input images $I_{-1}, I_{0}$, and $I_{+1}$ results in sharper images as evidenced by the Fourier spectra comparison between input in-focus image $I_{0}$ and the HUnet image. Frequency analysis suggests that HUnet images have a $46 \%$ wider frequency support (median value for all 7 images). Moreover, HUnet expectedly suppresses high-frequency noise and in-paints missing frequencies to achieve sharper nuclei boundaries. 
Varying network size for Znet. We investigated whether increasing the phase estimation network size from 14 to 19 million parameters could further improve results. Our results show the same overall performance. The larger network performance on the fourth order coefficients was slightly reduced in the fly dataset and marginally better on the zebrafish dataset with similar performance on the second and third order coefficients (Fig. 5). However the generalization of the larger network trained on zebrafish embryo images was reduced when applied to fly embryo images - suggesting over-fitting to dataset specific features (Table 3).

Table 1. HUnet performance and comparison with iterative non-blind deconvolution. PD stands for Phase Diversity.

\begin{tabular}{lcccc} 
& \multicolumn{2}{c}{ fly } & \multicolumn{2}{c}{ zebrafish } \\
& $r^{2}$ & SSIM & $r^{2}$ & SSIM \\
\hline HUnet With PD & $\mathbf{0 . 9 2 9}$ & $\mathbf{0 . 4 2 1}$ & $\mathbf{0 . 9 8 7}$ & $\mathbf{0 . 9 3 2}$ \\
HUnet No PD & 0.927 & 0.404 & 0.982 & 0.922 \\
\hline ICTM & 0.693 & 0.317 & 0.870 & 0.556 \\
BVLS & 0.662 & 0.294 & 0.856 & 0.512 \\
RLTV & 0.623 & 0.295 & 0.818 & 0.458 \\
\hline in-focus & 0.691 & 0.264 & 0.869 & 0.475
\end{tabular}

Table 2. HUnet generalisation between fly and zebrafish. Training dataset along rows and testing dataset along columns. Numbers are SSIM.

\begin{tabular}{lcc} 
& fly (test) & zebrafish (test) \\
\hline fly (train) & 0.421 & 0.727 \\
zebrafish (train) & 0.466 & 0.932
\end{tabular}

Table 3. Znet performance. Training dataset along rows and testing dataset along columns. Two Znet architectures with 14 and 19 million parameters where tested.

\begin{tabular}{cccccc} 
& & \multicolumn{2}{c}{ fly (test) } & \multicolumn{2}{c}{ zebrafish (test) } \\
\hline & & $r^{2}$ & MAE & $r^{2}$ & MAE \\
\hline $14 \mathrm{M}$ & fly & 0.739 & 0.134 & 0.791 & 0.124 \\
& zebrafish & 0.622 & 0.163 & 0.841 & 0.106 \\
\multirow{2}{*}{$19 \mathrm{M}$} & fly & 0.738 & 0.136 & 0.786 & 0.125 \\
& zebrafish & 0.576 & 0.176 & 0.838 & 0.107
\end{tabular}


To train jointly, or not. Unfortunately, we could not find a configuration in which joint training would significantly improve the performance of HUnet for both datasets. While it improves Znet performance slightly for zebrafish dataset, it reduces performance for fly dataset. Possible explanations are: (i) using a trained PSFnet instead of a differentiable analytical model is sub-optimal since a network can only approach and not attain the exactness of an analytical model, (ii) the consistency loss only refers to $I_{0}$ instead of to the whole phase diversity stack $I_{-1}$, $I_{0}$, and $I_{+1}$, which likely would provide stronger consistency information, (iii) the large mismatch between the number of parameters in HUnet and Znet (Table 4), (iv) finally, the non-ideal nature of self-consistency loss: the mean absolute error on blurred images might not be helpful. Overall, more work is needed to understand how and in which circumstances physics based consistency losses could help.

Table 4. Joint training performance. Training dataset along rows and testing dataset along columns.

\begin{tabular}{cccccc} 
& & \multicolumn{2}{c}{ fly (test) } & \multicolumn{2}{c}{ zebrafish (test) } \\
& & $r^{2}$ & SSIM & $r^{2}$ & SSIM \\
\hline \multirow{2}{*}{ HUnet } & in-focus & 0.691 & 0.264 & 0.869 & 0.475 \\
& fly & 0.925 & 0.416 & 0.971 & 0.750 \\
& zebra & 0.920 & 0.464 & 0.974 & 0.891 \\
\hline \multirow{2}{*}{ Znet } & & $r^{2}$ & MAE & $r^{2}$ & MAE \\
& fly & 0.688 & 0.148 & 0.742 & 0.140 \\
& zebra & 0.581 & 0.168 & 0.844 & 0.103
\end{tabular}

\section{Discussion}

We have shown on simulations that convolutional models can be trained to deconvolve (HUnet) and estimate aberrations (Znet) from simulated phase diverse acquisitions. Moreover, our experiments with images from a light-sheet microscope also confirm improved resolution (46\% wider frequency support) even in regions where assumptions made during training are not fully guaranteed - i.e. in the presence of confounding factors such as high-order aberrations, scattering and variance in the light-sheet thickness.

The most interesting observation is the robust performance of our deconvolution model (HUnet) in the absence of phase-diversity. This suggests that the model can directly be used for blind deconvolution of single images. How is this possible? Our interpretation is that the model captures an implicit prior of the highly stereotypical structure of fluorescently labelled nuclei and does not require phase information to be competitive. This suggests that such models can be made quite robust against uncertain aberration regimes as long as the aberrations are not too severe. Of course, such trained models are necessarily limited to the stereotypy of their training data.

In contrast, and as expected, phase estimation via Znet does require phase-diverse images as input. With the caveat that a direct comparison of models trained on different datasets is non-ideal, our phase estimation model outperforms the semi-blind model in [12]. Znet achieved a mean $r^{2}$ of 0.74 and 0.84 on fly and zebrafish datasets respectively for regressing astigmatism, coma and trefoil - numbers to be compared to a $r^{2}$ of 0.58 in [12] for regressing focus and 
astigmatism. The reduced performance on the fourth order coefficients could be attributed to potential aliasing of the PSF.

Future work could focus on technical aspects such as investigating how to improve performance with adversarial loss training, optimising the architecture for single image inputs, as well as improving our simulation model. Moreover, it is possible to replace PSFnet with a non-learned differentiable analytical function. More importantly, extending our framework in 3D would be key to be able to deconvolve acquisitions axially.

From an adaptive optics perspective, the strong performance of Znet suggests that it could be used as part of an online adaptive optics setup for the purpose of closed-loop wavefront estimation and correction. In this context, the advantage of using neural network inference is its speed compared to iterative schemes - it runs a single pass per input and is often hardware accelerated. However, it is unclear that defocus-only phase-diversity per se is the right approach due to the lack of contrast between the images within the stack. Another approach, perhaps involving complex phase relations like those in spiral phase masks, may provide better contrast [32], and would thus provide more robustness to noise and better performance for higher order aberrations.

From a light-sheet imaging perspective, our experimental evidence suggests that low-order optical aberrations are not the sole, and perhaps not the dominant, cause of image degradation. As we have shown, scattering on both illumination and detection paths offer considerable obstacles to methods that assume a thin light-sheet, low-order aberrations, and only ballistic photons. It would interesting to see what deep learning can do to address these issues.

\section{Acknowledgments}

We thank Dr. Jan Huisken from the Morgridge Institute for Research, Madison, Wisconsin, USA for kindly providing zebrafish transgenic lines (h2afva:h2afva-mCherry). Thanks to our Chan Zuckerberg Biohub colleagues Li-Hao Yeh and Shalin Metha for discussion, advice and feedback. Finally, we thank the Chan Zuckerberg Biohub and its donors for funding this work.

\section{References}

1. P. J. Keller, A. D. Schmidt, J. Wittbrodt, and E. H. Stelzer, "Reconstruction of zebrafish early embryonic development by scanned light sheet microscopy," science 322, 1065-1069 (2008).

2. L. A. Royer, W. C. Lemon, R. K. Chhetri, Y. Wan, M. Coleman, E. W. Myers, and P. J. Keller, "Adaptive light-sheet microscopy for long-term, high-resolution imaging in living organisms," Nat. Biotechnol. 34, 1267-1278 (2016).

3. N. Dey, L. Blanc-Féraud, C. Zimmer, P. Roux, Z. Kam, J.-C. Olivo-Marin, and J. Zerubia, "Richardson-lucy algorithm with total variation regularization for $3 \mathrm{~d}$ confocal microscope deconvolution." Microsc. research technique 69-4, 260-6 (2006).

4. S. Preibisch, F. Amat, E. Stamataki, M. Sarov, R. H. Singer, E. Myers, and P. Tomancak, "Efficient bayesian-based multiview deconvolution," Nat. methods 11, 645 (2014).

5. B. Schmid and J. Huisken, "Real-time multi-view deconvolution," Bioinformatics 31, 3398-3400 (2015).

6. D. Perrone and P. Favaro, "A clearer picture of total variation blind deconvolution," IEEE Transactions on Pattern Analysis Mach. Intell. 38, 1041-1055 (2016).

7. R. D. Simmonds and M. J. Booth, "Modelling of multi-conjugate adaptive optics for spatially variant aberrations in microscopy,” J. Opt. 15, 094010 (2013).

8. R. A. Gonsalves, "Phase retrieval and diversity in adaptive optics," Opt. Eng. 21, $21-21-4$ (1982).

9. R. G. Paxman, T. J. Schulz, and J. R. Fienup, "Joint estimation of object and aberrations by using phase diversity," JOSA A 9, 1072-1085 (1992).

10. C. Belthangady and L. A. Royer, "Applications, promises, and pitfalls of deep learning for fluorescence image reconstruction," Nat. methods p. 1 (2019).

11. J. Zhang, J. shan Pan, W.-S. Lai, R. W. H. Lau, and M.-H. Yang, "Learning fully convolutional networks for iterative non-blind deconvolution,” 2017 IEEE Conf. on Comput. Vis. Pattern Recognit. (CVPR) pp. 6969-6977 (2017).

12. A. Shajkofci and M. Liebling, "Semi-blind spatially-variant deconvolution in optical microscopy with local point spread function estimation by use of convolutional neural networks," in 2018 25th IEEE International Conference on Image Processing (ICIP), (2018), pp. 3818-3822.

13. C. J. Schuler, M. Hirsch, S. Harmeling, and B. Schölkopf, "Learning to deblur," in NIPS 2014 Deep Learning and Representation Learning Workshop, (2014).

14. S. W. Paine and J. R. Fienup, "Machine learning for improved image-based wavefront sensing," Opt. Lett. 43, 1235-1238 (2018). 
15. Y. Nishizaki, M. Valdivia, R. Horisaki, K. Kitaguchi, M. Saito, J. Tanida, and E. Vera, "Deep learning wavefront sensing," Opt. Express 27, 240-251 (2019).

16. A. Bhatia and E. Wolf, "The zernike circle polynomials occurring in diffraction theory," Proc. Phys. Soc. Sect. B 65, 909 (1952).

17. R. G. Paxman, T. J. Schulz, and J. R. Fienup, "Joint estimation of object and aberrations by using phase diversity," JOSA A 9, 1072-1085 (1992).

18. M. Weigert, U. Schmidt, T. Boothe, A. Müller, A. Dibrov, A. Jain, B. Wilhelm, D. Schmidt, C. Broaddus, S. Culley et al., "Content-aware image restoration: pushing the limits of fluorescence microscopy," Nat. methods 15, 1090 (2018).

19. J. Batson and L. Royer, "Noise2self: Blind denoising by self-supervision," in International Conference on Machine Learning, (2019), pp. 524-533.

20. S. J. Yang, M. Berndl, D. Michael Ando, M. Barch, A. Narayanaswamy, E. Christiansen, S. Hoyer, C. Roat, J. Hung, C. T. Rueden, A. Shankar, S. Finkbeiner, and P. Nelson, "Assessing microscope image focus quality with deep learning," BMC Bioinforma. 19, 77 (2018).

21. O. Ronneberger, P.Fischer, and T. Brox, "U-net: Convolutional networks for biomedical image segmentation," in Medical Image Computing and Computer-Assisted Intervention (MICCAI), vol. 9351 of LNCS (2015), pp. 234-241.

22. M. L. C. S. Radford, A., "Unsupervised representation learning with deep convolutional generative adversarial networks," CoRR abs/1511.06434 (2015).

23. O. Ronneberger, P. Fischer, and T. Brox, "U-net: Convolutional networks for biomedical image segmentation," in International Conference on Medical image computing and computer-assisted intervention, (Springer, 2015), pp. 234-241.

24. W. H. Richardson, "Bayesian-based iterative method of image restoration," JoSA 62, 55-59 (1972).

25. N. Dey, L. Blanc-Féraud, C. Zimmer, Z. Kam, J.-C. Olivo-Marin, and J. Zerubia, "A deconvolution method for confocal microscopy with total variation regularization," in 2004 2nd IEEE International Symposium on Biomedical Imaging: Nano to Macro (IEEE Cat No. 04EX821), (IEEE, 2004), pp. 1223-1226.

26. V. Kempen and V. Vliet, "The influence of the regularization parameter and the first estimate on the performance of tikhonov regularized non-linear image restoration algorithms," J. Microsc. 198, 63-75 (2000).

27. D. Sage, L. Donati, F. Soulez, D. Fortun, G. Schmit, A. Seitz, R. Guiet, C. Vonesch, and M. Unser, "Deconvolutionlab2: An open-source software for deconvolution microscopy," Methods 115, 28 - 41 (2017). Image Processing for Biologists.

28. Z. Wang, A. C. Bovik, H. R. Sheikh, E. P. Simoncelli et al., "Image quality assessment: from error visibility to structural similarity," IEEE transactions on image processing 13, 600-612 (2004).

29. R. Mizutani, R. Saiga, S. Takekoshi, C. Inomoto, N. Nakamura, M. Itokawa, M. Arai, K. Oshima, A. Takeuchi, K. Uesugi et al., "A method for estimating spatial resolution of real image in the fourier domain," J. microscopy 261, 57-66 (2016).

30. T.-1. Liu, S. Upadhyayula, D. E. Milkie, V. Singh, K. Wang, I. A. Swinburne, K. R. Mosaliganti, Z. M. Collins, T. W. Hiscock, J. Shea et al., "Observing the cell in its native state: Imaging subcellular dynamics in multicellular organisms," Science 360, eaaq1392 (2018).

31. M. Weigert, L. Royer, F. Jug, and G. Myers, "Isotropic reconstruction of 3d fluorescence microscopy images using convolutional neural networks," in International Conference on Medical Image Computing and Computer-Assisted Intervention, (Springer, 2017), pp. 126-134.

32. M. K. Sharma, C. Gaur, P. Senthilkumaran, and K. Khare, "Phase imaging using spiral-phase diversity," Appl. Opt. 54, 3979-3985 (2015). 